\title{
Some Instructive Mathematical Errors
}

\author{
RICHARD P. BRENT, Mathematical Sciences Institute, Australian National University, Australia
}

\begin{abstract}
We describe various errors in the mathematical literature, and consider how some of them might have been avoided, or at least detected at an earlier stage, using tools such as Maple or Sage. Our examples are drawn from three broad categories of errors. First, we consider some significant errors made by highly-regarded mathematicians. In some cases these errors were not detected until many years after their publication. Second, we consider in some detail an error that was recently detected by the author. This error in a refereed journal led to further errors by at least one author who relied on the (incorrect) result. Finally, we mention some instructive errors that have been detected in the author's own published papers.
\end{abstract}

CCS Concepts: • Mathematics of computing $\rightarrow$ Number-theoretic computations; $\bullet$ Computing methodologies $\rightarrow$ Hybrid symbolic-numeric methods;

Additional Key Words and Phrases: mathematical errors, four-colour theorem, abc conjecture, chaos, prime zeta function, Riemann hypothesis, GRH, analysis of algorithms, Mellin transforms, arithmetic-geometric mean

\section{Recommended Reference Format:}

Richard P. Brent. 2021. Some Instructive Mathematical Errors . Maple Trans. 1, 1, Article 14069 (July 2021), 16 pages. https://doi.org/10.5206/mt.v1i1.14069

\section{Introduction}

Those that fail to learn from history are doomed to repeat it.

\section{Winston Churchill [23].}

Since mathematics is a human endeavour, errors can and do occur. It is worth studying past errors and learning from them to reduce the likelihood of making similar errors in the future.

We are concerned with nontrivial errors made by working mathematicians, not with the class of errors that school-children often make in their homework.

The errors that we consider can be grouped into three broad categories.

(1) Well-known errors made by prominent mathematicians (see §2).

(2) Errors discovered by the author in other mathematicians' work (§3).

(3) Some errors in, or relevant to, the author's own work (\$4).

It is worth mentioning the errors in category 1 in order to show that even the best mathematicians are fallible. Also, correcting an error may lead to interesting mathematics (see for example $\S 2.3$ and $\S 2.5)$. Our list is by no means exhaustive.

Errors in category 2 were discovered recently by the author. These errors are discussed in some detail in order to show how they were detected, and how they might have been avoided.

Errors in category 3 are described as a penance, and because the reader may find them instructive.

Our definition of "error" is rather broad. An error in a proof might be an unjustified assumption, or a gap that needs to be filled. As well as errors in published or submitted papers, we include some incorrect results that were announced in other ways, some implicit errors (see §4.3), and some claims that were expressed as conjectures rather than theorems (see $§ 2.2, \S 4.2$ ).

Author's address: Richard P. Brent, Mathematical Sciences Institute, Australian National University, Canberra, Australia.

Permission to make digital or hard copies of all or part of this work for any use is granted without fee, provided that copies bear this notice and the full citation on the first page. Copyrights for third-party components of this work must be honored. (C) 2021 Copyright held by the owner/author(s). Publication rights licensed to Maple Transactions, under Creative Commons CC-BY 4.0 License.

https://doi.org/10.5206/mt.v1i1.14069

Maple Trans., Vol. 1, No. 1, Article 14069. Publication date: July 2021. 


\section{Some well-known errors}

Many errors have been made by highly-regarded mathematicians. They have generally been welldocumented, so we merely give an overview, with references to further information. Our list is (approximately) in chronological order. A considerably longer list is available online [70].

\subsection{The four-colour theorem}

Many fallacious proofs of the four-colour theorem have been given. Notable are two, by Kempe [37] (1879) and Tait [61] (1880), that both stood for 11 years before the errors were noticed [68]. The first correct proof, by Appel and Haken [3] in 1976, used some of Kempe's ideas. The Appel-Haken proof was at first controversial, because it depended on the computer-aided verification of 1834 "reducible configurations" which could not feasibly be verified by hand. In fact, some relatively minor errors were discovered later, and corrected in the 1989 book by Appel and Haken [4]. There is still no known "simple" proof that does not involve checking a large number of cases.

\subsection{Mertens and Stieltjes}

In 1897, Franz Mertens [45] conjectured, on the basis of numerical evidence, that $|M(x)| \leqslant x^{1 / 2}$ for all $x>1$, where $M(x):=\sum_{n \leqslant x} \mu(n)$, and $\mu(n)$ is the Möbius function. The same inequality was also conjectured (in 1885) by Thomas Stieltjes, in a letter to Charles Hermite [60,71].

In the same letter to Hermite, Stieltjes claimed to have a proof of the weaker result that $M(x)=$ $O\left(x^{1 / 2}\right)$. However, he never published a proof, and none was found in his papers after his death.

It is well-known $[63, \S 14.28]$ that $M(x)=O\left(x^{1 / 2+\varepsilon}\right)$ for all $\varepsilon>0$ if and only if the Riemann Hypothesis (RH) is true. Thus, the claimed result of Stieltjes would imply RH. In fact, it would imply even more.

In 1985, Andrew Odlyzko and Herman te Riele [50] disproved the conjecture of Mertens. The disproof was numerical, and did not disprove the claim of Stieltjes. However, for reasons given in [50, §§1-2], most experts believe that the claim $M(x)=O\left(x^{1 / 2}\right)$ of Stieltjes is false.

\subsection{Poincaré’s prize essay}

In 1888, Henri Poincaré submitted a paper, entitled The Three-Body Problem and the Equations of Dynamics, to a competition sponsored by King Oscar II of Sweden and Norway, and the journal Acta Mathematica (edited by Gösta Mittag-Leffler). The prize committee (Hermite, Mittag-Leffler, and Weierstrass) awarded Poincaré the prize. The committee stated:

It is the deep and original work of a mathematical genius whose position is among the greatest mathematicians of the century. The most important and difficult questions, like the stability of the world system, are treated using methods which open a new era in celestial mechanics.

While Poincaré's manuscript was being prepared for printing in Acta Mathematica, sub-editor Edvard Phragmén communicated with Poincaré about parts of the manuscript that he found difficult to understand.

If the author were not what he is, I would not for a

moment hesitate to say that he has made a great mistake here.

$$
\text { Phragmén, December 1888, see [48]. }
$$

Eventually, in December 1889, Poincaré admitted that he had made an error with a critical consequence - his claimed proof of the stability of the solar system was invalid! Unfortunately, the paper [53] had already been printed, and Mittag-Leffler had started to distribute it. With some 
difficulty, Mittag-Leffler managed to recall almost all of the copies. For further details and references, see [31].

Poincaré prepared a corrected version, about twice as long as the original prize entry, and it was eventually published [54]. Poincaré had to pay the extra costs involved, which exceeded the prize money that he had won.

The story had a happy ending as, in realising his error and making his corrections, Poincaré discovered the phenomenon of chaos [7, 25, 32, 43, 49]. Some of the credit for this great discovery must go to Phragmén.

\subsection{Rademacher and the Riemann Hypothesis}

If you want to climb the Matterhorn you might first wish to go to Zermatt where those who have tried are buried.

$$
\text { George Pólya }{ }^{1}
$$

In 1943, Hans Rademacher [8] submitted a proof ${ }^{2}$ of the Riemann Hypothesis (RH) to Transactions of the American Mathematical Society. It was accepted and was scheduled to appear in the May 1945 issue. At the last moment, Rademacher withdrew the paper, because Carl Siegel had found a flaw in his reasoning. The paper did not appear in the Transactions, but the story was told to a wide audience in Time magazine [2].

Since Rademacher's manuscript is not available, we can not say what his error was. However, there have been suggestions (see e.g. [42]) that he used a proof by contradiction. In other words, he assumed the existence of a zero $\rho$ of $\zeta(s)$ with $\mathfrak{R}(\rho)>1 / 2$, and from this assumption he derived a contradiction, implying that $\mathfrak{R}(\rho) \leqslant 1 / 2$. This would prove $\mathrm{RH}$. However, in any proof by contradiction, one has to be extremely careful, as an error in the proof might lead to an erroneous contradiction.

We remark that several other mathematicians have claimed to prove RH. Some serious attempts are mentioned in [12, Ch. 8]. Regarding "amateur" attempts, Roger Heath-Brown (quoted in [55, pg. 112]) comments:

I receive unsolicited manuscripts quite frequently - one finds a particular person who has an idea, and no matter how many times you point out a mistake, they correct that and produce something else that is also a mistake.

\subsection{Wiles and the proof of Fermat's Last Theorem}

What we now call Fermat's Last Theorem (FLT) was mentioned by Pierre de Fermat around 1637 in the margin of his copy of Diophantus's Arithmetica. The story is too well-known to warrant repeating here.

There have been a great many erroneous proofs of FLT, see [67]. The list might well include Fermat's proof, referred to in his marginal note - we will never know with certainty, but in view of developments over the next 350 years, it seems very unlikely that his proof was correct.

In June 1993, Andrew Wiles announced a proof of the Taniyama-Shimura conjecture for semistable elliptic curves. By previous work of Ribet and of Frey (building on earlier work by other mathematicians), this was known to imply FLT.

Wiles presented his proof at a series of lectures (in Cambridge, UK), and submitted a paper to Annals of Mathematics. However, while refereeing the paper, Nick Katz found a gap in the proof.

\footnotetext{
${ }^{1}$ Quoted by Lars Hörmander, see [12, pg. 69].

${ }^{2}$ Time [2] says "disproving the Riemann Hypothesis", but this is likely to be an error, see [42].
} 
Wiles worked to repair his proof, first alone, and then with his former student Richard Taylor. By September 1994 they were almost ready to admit defeat. Then, while trying to understand why his approach could not be made to work, Wiles had a sudden insight.

I was sitting at my desk examining the Kolyvagin-Flach method. It wasn't that I believed I could make it work, but I thought that at least I could explain why it didn't work. Suddenly I had this incredible revelation. I realised that, the Kolyvagin-Flach method wasn't working, but it was all I needed to make my original Iwasawa theory work from three years earlier. So out of the ashes of Kolyvagin-Flach seemed to rise the true answer to the problem. It was so indescribably beautiful; it was so simple and so elegant.

Andrew Wiles, quoted by Simon Singh [59].

Wiles's insight led to a corrected proof of FLT, published in 1995 as two papers, one by Wiles [74], and a companion paper by Taylor and Wiles [62]. For a non-technical overview, see [59, 73].

Table 1. Comparing the sagas of Poincaré and Wiles

\begin{tabular}{|c|c|c|}
\hline Author & Poincaré & Wiles \\
\hline Topic & Three-body problem & FLT \\
\hline Date & $1888-1892$ & 1993-1995 \\
\hline Error(s) found by & Phragmén & Katz \\
\hline Corrected by & Poincaré & Wiles and Taylor \\
\hline $\begin{array}{l}\text { Some further } \\
\text { developments }\end{array}$ & $\begin{array}{l}\text { Theory of chaos in } \\
\text { dynamical systems } \\
\text { developed by Poincaré } \\
\text { and later authors }\end{array}$ & $\begin{array}{l}\text { Modularity theorem } \\
\text { proved by Breuil, } \\
\text { Conrad, Diamond, } \\
\text { and Taylor (2001) }\end{array}$ \\
\hline
\end{tabular}

The story (or perhaps we can justifiably call it a saga) of Wiles's proof of FLT has some striking analogies to the story/saga of Poincaré’s prize essay (§2.3). Wiles’s original manuscript had an error (discovered by Katz); Poincaré's had errors (discovered by Phragmén). Although both papers included other results that were of value, the errors invalidated their main results. The errors were corrected, though not without difficulty, and the corrected results were published and have been accepted as valid. Both papers led to further developments: in the case of Wiles, proof of the Taniyama-Shimura-Weil conjecture for all elliptic curves (a result now known as the modularity theorem) by Breuil, Conrad, Diamond, and Taylor [21]; in the case of Poincaré, the development of chaos theory by Poincaré and many subsequent authors. The analogies are summarised in Table 1.

\subsection{Mochizuki's claimed proof of the abc conjecture}

The $a b c$ conjecture ${ }^{3}$ considers three relatively prime positive integers $a, b, c$ such that $a+b=c$. Let $d$ be the product of the distinct prime factors of $a b c$ (hence the name " $a b c$ conjecture"). The conjecture gives a lower bound on $d$ that (conjecturely) holds for all but a finite number of cases. ${ }^{4}$

The $a b c$ conjecture was shown by Oesterlé [51] to be essentially equivalent to the Szpiro conjecture, which relates the conductor and the discriminant of an elliptic curve. If true, these (equivalent)

\footnotetext{
${ }^{3}$ Also, perhaps more informatively, known as the Oesterlé-Masser conjecture after its proposers David Masser [40] and Joseph Oesterlé [51].

${ }^{4}$ The bound on the finite number $N(\varepsilon)$ of exceptional cases depends on a positive but arbitrarily small parameter $\varepsilon$; for details see [66].
} 
conjectures would have many interesting consequences. Some of these consequences are already known to be true, e.g. Roth's theorem and the theorem of Faltings (formerly known as the Mordell conjecture); others still have the status of conjectures, e.g. the Fermat-Catalan conjecture, which is a generalisation of FLT.

In August 2012, Shinichi Mochizuki claimed a proof of Szpiro's conjecture (and hence the $a b c$ conjecture) by developing a new theory called inter-universal Teichmüller theory (IUTT) [6]. However, the proof has not been generally accepted by the mathematical community [72]. On one side we have views such as:

We, the authors of this note, came to the conclusion that there is no proof. We are going to explain where, in our opinion, the suggested proof has a problem, a problem so severe that in our opinion small modifications will not rescue the proof strategy. We supplement our report by mentioning dissenting views from Prof. Mochizuki and Prof. Hoshi about the issues we raise with the proof and whether it constitutes a gap at all.

Peter Scholze and Jakob Stix [58].

On the other side, Mochizuki still claims that his proof is correct, and has published it in a refereed journal [22]. Mochizuki was at that time the editor-in-chief of the journal. ${ }^{5}$

At the present time, all that we can say with certainty is that the status of Mochizuki's proof is unclear. For further information, see [38, 58, 75], and comments on MathOverflow [41].

\subsection{Summary of the examples above}

A new scientific truth does not triumph by convincing its opponents and making them see the light, but rather because its opponents eventually die and a new generation grows up that is familiar with it.

\section{Max Planck [52]}

We have given examples of errors that were found and corrected, see §2.3 (Poincaré) and §2.5 (Wiles). Also, we have mentioned some proofs that were fatally flawed, but a different (correct) proof of the result was found later, see $\$ 2.1$ (Kempe, Tait, Appel and Haken). In other cases an error was found and acknowledged by the author, and no correct proof of the result is known, see $§ 2.4$ (Rademacher).

Yet another category is where a proof is unlikely to be correct, but this is impossible to verify, as the author has died and the claimed proof was never written down (or has been lost), see $§ 2.2$ (Stieltjes). Fermat's claimed proof (of FLT) is in this category.

Finally, we have given one example of a proof that is disputed, in that the author maintains that it is correct, but a significant number of experts disagree, see §2.6. In such cases, time will tell eventually the proof will be accepted as correct, ${ }^{6}$ or the author and his supporters will die and the proof will be relegated to "the dustbin of history".

\section{Agélas and Vassilev-Missana}

In March 2021, Léo Agélas sent the author a preprint that had appeared online [1] and had also been submitted to a journal. In [1], Agélas states ${ }^{7}$

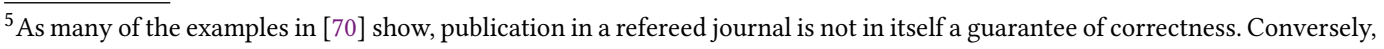
some important and correct papers were never published in refereed journals - one example is the work of Perelman on the Poincaré conjecture, which solved one of the Millennium Prize Problems, but was only published on the preprint server arXiv.

${ }^{6}$ Possibly after being rewritten to fill in gaps and make it more readily comprehensible.

${ }^{7}$ We use the word "Claim" for a statement that we may later prove to be false, in order to distinguish it from a statement that we believe to be true.
} 
Claim 1 (Agélas, Theorem 2.1). For any Dirichlet character $\chi$ modulo $k$, the Dirichlet L-function $L(\chi, s)$ has all its non-trivial zeros on the critical line $\mathfrak{R}(s)=\frac{1}{2}$.

This is the Generalized Riemann Hypothesis (GRH), probably formulated by Adolf Piltz in 1884 (see Davenport [24, p. 124]). A special case, which corresponds to the principal character $\chi_{0}(n)=1$ and the Riemann zeta-function $\zeta(s)$, is the Riemann Hypothesis, cf. §2.4.

Agélas defines the half-plane $\mathcal{A}:=\{s \in \mathbb{C}: \mathfrak{R}(s)>1\}$, and two Dirichlet series (convergent for $s \in \mathcal{A}):$

$$
P(\chi, s):=\sum_{p \in \mathcal{P}} \chi(p) p^{-s}
$$

and

$$
P_{2}(\chi, s):=\sum_{p \in \mathcal{P}} \chi(p)^{2} p^{-s},
$$

where $\mathcal{P}$ is the set of primes $\{2,3,5, \ldots\}$.

When trying to understand the proof of Claim 1 by Agélas, we considered the case of the Riemann zeta-function. Since this was sufficient to find an error in the proof, we only need to consider this case. Thus we can take $\chi(p)=1$, so $P(\chi, s)$ and $P_{2}(\chi, s)$ both reduce to the usual prime zeta function [30]

$$
P(s):=\sum_{p \in \mathcal{P}} p^{-s}
$$

It is well-known [30, p. 188] that, for $\mathfrak{R}(s)>1$,

$$
P(s)=\sum_{k=1}^{\infty} \frac{\mu(k)}{k} \log \zeta(k s) .
$$

Vassilev-Missana [65] states

Claim 2 (Vassilev-Missana, Theorem 1). For integer $s>1$, the relation

$$
(1-P(s))^{2}=\frac{2}{\zeta(s)}-1+P(2 s) \text { holds. }
$$

Agélas states

Lemma 2.3 appears as an extension of Theorem 1 of Vassilev-Missana (2016), we give here the details of the proof as it is at the heart of the Theorem obtained in this paper. For this, we borrow the arguments used in Vassilev-Missana (2016).

He then states

Claim 3 (Agélas, Lemma 2.3). For $s \in \mathcal{A}$, we have

$$
(1-P(\chi, s))^{2} L(\chi, s)-\left(P_{2}(\chi, 2 s)-1\right) L(\chi, s)=2 .
$$

In the case that we consider, namely $L(\chi, s)=\zeta(s)$, both Claim 2 and Claim 3 amount to the same relation, which we can write in an equivalent form as

$$
\frac{2}{\zeta(s)}=2-2 P(s)+(P(s))^{2}-P(2 s) .
$$

In $\S 3.1$ we show that (2) is false. This implies that Lemma 2.3 of Agélas is false, as is Theorem 1 of Vassilev-Missana. Theorem 2.1 of Agélas (the GRH) may be true, but has not been proved. Theorem 2 of Vassilev-Missana is false, as shown in §3.2.

\footnotetext{
${ }^{8}$ It is not clear why Vassilev-Missana imposes such a strong restriction on $s$; we might expect the relation to hold for all $s \in \mathcal{A}$, or even (using analytic continuation) for almost all $s \in\{z \in \mathbb{C}: \mathfrak{R}(z)>0\}$.
} 


\subsection{Disproving claim (2)}

Five methods to disprove (2) are given in [18]. We give three of them here. The methods that are omitted here involve analytic continuation into the strip $0<\mathfrak{R} s \leqslant 1$.

Method 1. Expand each side of (2) as a Dirichlet series $\sum a_{n} n^{-s}$. On the right-hand side (RHS), the only terms with nonzero coefficients $a_{n}$ are for integers $n$ of the form $p^{\alpha} q^{\beta}$, where $p$ and $q$ are primes, $\alpha \geqslant 0$, and $\beta \geqslant 0$. However, on the left-hand side (LHS), we find $a_{30}=-2 \neq 0$, since $30=2 \times 3 \times 5$ has three distinct prime factors, implying that $\mu(30)=-1$. By the uniqueness of Dirichlet series that converge absolutely for all sufficiently large values of $\mathfrak{R}(s)$ [34, Thm. 4.8], we have a contradiction, so (2) is false.

Remark 1. Instead of 30 we could take any squarefree positive integer with more than two prime factors.

Method 2. We can evaluate both sides of (2) numerically for one or more convenient values of $s$. If we take $s=2 k$ for some positive integer $k$, then the LHS of (2) can easily be evaluated using Euler's formula

$$
\zeta(2 k)=\frac{(-1)^{k-1}(2 \pi)^{2 k}}{2 \cdot(2 k) !} B_{2 k},
$$

where $B_{2 k}$ is a Bernoulli number. The RHS can be evaluated by using (1). Taking $k=1$, i.e. $s=2$, the LHS is $12 / \pi^{2}=1.2158542$ and the RHS is 1.2230397 (both values correct to 7 decimals). Thus, $\mid$ LHS - RHS $\mid>0.007$. This is a contradiction, so (2) is false.

Remark 2. It is always a good idea to verify identities numerically whenever it is convenient to do so. A surprising number of typographical and more serious errors can be found in this manner. Early mathematicians such as Euler, Gauss, and Riemann were well aware of the value of numerical computation, even though they lacked the electronic tools and mathematical software (such as Maple, Magma, Mathematica, SAGE, ...) that are available today.

If we had followed the philosophy of experimental mathematics [9], we would have attempted method 2 first. However, method 1 has the advantage that all computations are easy to do by hand (or mental arithmetic). Method 2 is slightly more work, as it requires writing a small program.

Method 3. We consider the behaviour of each side of (2) near $s=1$. On the LHS there is a simple zero at $s=1$, since the denominator $\zeta(s)$ has a simple pole. On the RHS there is a logarithmic singularity of the form $a(\log (s-1))^{2}+b \log (s-1)+O(1)$. This is a contradiction, so (2) is false.

\subsection{Theorem 2 of Vassilev-Missana is false}

Vassilev-Missana [65, Theorem 2] makes the following claim.

Claim 4. For integer $s>1$,

$$
P(s)=1-\sqrt{2 / \zeta(s)-\sqrt{2 / \zeta(2 s)-\sqrt{2 / \zeta(4 s)-\sqrt{2 / \zeta(8 s)-\cdots}}}}
$$

Proof that Claim 4 is incorrect. Assume that Claim 4 is correct. Replacing $s$ by $2 s$ and using the result to simplify (3), we obtain

$$
1-P(s)=\sqrt{2 / \zeta(s)-(1-P(2 s))} .
$$

Squaring both sides of (4) and simplifying gives (2), but we showed in $§ 3.1$ that (2) is incorrect. This contradiction shows that Claim 4 is incorrect. 
Remark 3. An alternative is to resort to a variation on method 2 above. With $s=2$ we find numerically that $P(s) \approx 0.4522$ and

$$
1-\sqrt{2 / \zeta(s)-\sqrt{2 / \zeta(2 s)-\sqrt{2 / \zeta(4 s)-\cdots}}} \approx 0.4588 \neq P(s)
$$

where the numerical values are correct to 4 decimal places. Thus, (3) is incorrect.

Remark 4. It may not be clear what the infinite expression on the RHS of (3) means. We state Claim 4 more precisely as

$$
P(s)=1-\lim _{n \rightarrow \infty} \sqrt{2 / \zeta(s)-\sqrt{2 / \zeta(2 s)-\sqrt{2 / \zeta(4 s)-\cdots \sqrt{2 / \zeta\left(2^{n} s\right)}}}} .
$$

The limit exists and is real if $s$ is real, positive, and sufficiently large.

To evaluate (5) numerically, we could start with a sufficiently large value of $n$, then evaluate the nested square roots in (5) by working from right to left, using the values of $\zeta\left(2^{n} s\right), \zeta\left(2^{n-1} s\right), \ldots$, $\zeta(2 s), \zeta(s)$. In fact, it is desirable to replace $2 / \zeta\left(2^{n} s\right)$ in $(5)$ by $2 / \zeta\left(2^{n} s\right)-1$, as this gives the same limit but with faster convergence (for details see [18]).

\section{Errors in, or relevant to, the author's own work}

In this section we discuss three of the author's papers. The first, concerned with the analysis of the binary Euclidean algorithm, had some significant errors which were only noticed (and corrected) 21 years after the paper was published, although the paper had been referred to several times in the intervening period.

The second paper (§4.2), concerning integer multiplication, contained a conjecture which, although believed by the authors and supported by numerical evidence, was false. This was shown by Erdős in a 1960 paper [26] that, unfortunately, was written in Russian and difficult to access. Moreover, it turned out that Erdős's proof was incorrect, and was only corrected by Erdős and Sárközy some 20 years later [27].

The third paper (§4.3) pointed out an (incorrect) implicit assumption made in several papers, by various authors, concerning fast algorithms based on the arithmetic-geometric mean.

\subsection{Analysis of the binary Euclidean algorithm}

\section{Tam complicatæ evadunt, ut nulla spes superesse videatur. ${ }^{9}$}

\section{Gauss, notebook, 1800}

My 1976 paper [14] proposed a heuristic probabilistic model for the binary Euclidean algorithm. Some forty years later, the heuristic assumptions of the model were fully justified by Ian Morris [47], building on earlier work by Brigitte Vallée [64] and Gérard Maze [44]. The paper [14] contained some significant errors which were not noticed until 1997, when Donald Knuth was revising volume 2 of his classic series The Art of Computer Programming in preparation for publication of the third edition [39]. The errors all take the same form, which we illustrate by considering a typical case. Further details are given in [15, §9].

It is convenient to define $\lg x:=\log _{2} x=(\ln x) / \ln 2$. Consider the function $f:[0, \infty) \mapsto(0, \infty)$ defined by

$$
f(x):=\sum_{k=1}^{\infty} \frac{2^{-k}}{1+2^{k} x}
$$

\footnotetext{
${ }^{9}$ They come out so complicated that no hope appears to be left (Gauss referring to his analysis of the Euclidean algorithm).
} 
Observe that

$$
f^{\prime}(x)=-\sum_{k=1}^{\infty}\left(\frac{1}{1+2^{k} x}\right)^{2}
$$

where the series converges for all $x>0$, and as $x \rightarrow 0+$ we have $f^{\prime}(x) \sim \lg x$. Thus, we might expect $f(x)$ to have a logarithmic singularity of the form $x \lg x$ at the origin (though this turns out to be incorrect, see below).

In [14, Lemma 3.1], it is claimed that (with $f$ denoted by $D_{1}$ in [14]),

$$
f(x)=1+x \lg x+\frac{x}{2}-\frac{x^{2}}{1+x}+\sum_{k=1}^{\infty} \frac{(-1)^{k} x^{k+1}}{2^{k}-1}
$$

for $0<x<2$, but this is incorrect, as we shall show.

It was pointed out by Flajolet and Vallée [personal communication, 1997] that we can obtain an equivalent expression for $f(x)$ using Mellin transforms [28, App. B.7]. The Mellin transform of $g(x):=1 /(1+x)$ is

$$
g^{*}(s)=\int_{0}^{\infty} g(x) x^{s-1} d x=\frac{\pi}{\sin \pi s}
$$

in the strip $0<\mathfrak{R} s<1$. Now $f(x)=\sum_{k \geqslant 1} 2^{-k} g\left(2^{k} x\right)$, so the Mellin transform of $f(x)$ is

$$
f^{*}(s)=\sum_{k=1}^{\infty} 2^{-k(s+1)} g^{*}(s)=\frac{g^{*}(s)}{2^{s+1}-1} .
$$

Using the inverse Mellin transform, we can write $f(x)$ as a sum of residues of

$$
h(s):=\left(\frac{\pi}{\sin \pi s}\right) \frac{x^{-s}}{2^{s+1}-1}
$$

for $\mathfrak{R} s \leqslant 0$. The function $h(s)$ has poles for $s \in \mathbb{Z}$ (where $\sin \pi s=0$ ), and also for $s=-1+2 \pi i n / \ln 2$, $n \in \mathbb{Z}$ (where $2^{s+1}=1$ ). Note that there is a double pole at $s=-1$. Evaluating the residues gives, for $x \in(0,1)$,

$$
f(x)=1+x \lg x+\frac{x}{2}-\frac{x^{2}}{1+x}+\sum_{k=1}^{\infty} \frac{(-1)^{k} x^{k+1}}{2^{k}-1}+x P(\lg x)
$$

where

$$
P(t)=\frac{2 \pi}{\ln 2} \sum_{n=1}^{\infty} \frac{\sin 2 n \pi t}{\sinh \left(2 n \pi^{2} / \ln 2\right)}
$$

is a small periodic function arising from the non-real poles of $h(s)$.

We observe that the correct expression (8) differs from the incorrect (7) precisely by the addition of the small term $x P(\lg x)$.

The reason for the error in the proof of Lemma 3.1 of [14] is that it was simply assumed that $f(x)$ could be written as $\gamma(x) \lg (x)+\delta(x)$, where $\gamma(x)$ and $\delta(x)$ are analytic and regular in the unit disk $|x|<1$. Expressions for $\gamma(x)$ and $\delta(x)$ were then deduced, giving (7). The error was not in the deduction of (7), but in the incorrect assumption regarding the form of $f(x)$.

In retrospect, it should have been obvious that, if $x$ is regarded as a complex variable, then (6) defines an analytic function with poles at $x=-2^{-k}$ for $k=1,2,3, \ldots$ On the other hand, $\gamma(x) \lg (x)+$ $\delta(x)$ has only one singularity in the disk $|x|<1$, and that is the logarithmic singularity at $x=0$. Thus, as in the example of §3.1, the singularities differ.

The reader may ask why we did not follow our own advice (see Remark 2 in §3.1) and attempt to verify (7) numerically. In fact, we did verify the equality using floating point arithmetic on 
the computer available to us at the time (1976). ${ }^{10}$ This was insufficient to show a discrepancy, because $|P(t)|<7.8 \times 10^{-12}$ for $t \in \mathbb{R}$. The reason why $P(t)$ is so small is that the denominators $\sinh \left(2 n \pi^{2} / \ln 2\right)$ in $(9)$ are large; the smallest denominator is $\sinh \left(2 \pi^{2} / \ln 2\right)>1.16 \times 10^{12}$. Nowadays, we would attempt a verification to much higher precision (say 40 decimals), and this would be sufficient to detect the discrepancy caused by the term $x P(\lg x)$ in (8).

The analysis of the binary Euclidean algorithm predicts that the expected number of iterations is $\sim K \lg n$ for uniformly distributed $n$-bit inputs. Here $K$ is a constant that can be expressed as an integral, where the integrand includes a term involving $P(t)$. In 1997, Knuth attempted to evaluate $K$ accurately, and obtained

but I had found

$$
\begin{aligned}
& K=0.7059712461019 \underline{45} \cdots, \\
& K=0.7059712461019 \underline{16} \cdots .
\end{aligned}
$$

In a curious twist, it turned out that Knuth's value was incorrect, because he relied on some of the incorrect results in my paper [14], whereas my value was correct, because I had used a more direct numerical method that depended only on recurrences for certain distribution functions that were given correctly in [14]. With assistance from Flajolet and Vallée, we reached agreement on the correct value of $K$ just in time to meet the deadline for the third edition of [39].

\subsection{The Brent-Kung multiplication paper}

If you only want him to be able to cope with addition and subtraction, then any French or German university will do. But if you are intent on your son going on to multiplication and division - assuming that he has sufficient gifts - then you will have to send him to Italy.

15-th century advice, quoted by Georges Ifrah [35, pg. 577].

In a 1982 paper [19] with $H$. T. Kung, we considered the area $A$ and/or time $T$ required to perform multiplication of $n$-bit integers expressed in binary notation. We showed that, in a certain realistic model of computation, there is an area-time tradeoff, and $A T=\Omega\left(n^{3 / 2}\right)$. To obtain this bound we needed a lower bound on the function $M(N)$ defined by ${ }^{11}$

$$
M(N):=|\{i j \mid 0 \leqslant i<N, 0 \leqslant j<N\}| .
$$

Remark 5. In the number theory literature, it is customary to define

$$
M(N):=|\{i j \mid 0<i \leqslant N, 0<j \leqslant N\}|,
$$

which may be interpreted as the number of distinct entries in an $N \times N$ multiplication table. However, when considering $n$-bit binary multiplication, the definition (10) (with $N=2^{n}$ ) is more natural. Either definition can be used; it makes no difference to the asymptotics.

For our purposes, it was sufficient to use the easy lower bound ${ }^{12}$

$$
M(N) \geqslant \frac{N^{2}}{2 \log N} \text { for all } N \geqslant 4
$$

\footnotetext{
${ }^{10}$ We used a Univac 1100/42 mainframe with a 36-bit wordlength, and 27 bits for the floating-point fraction, equivalent to about 8 decimals. This was in the days before personal computers or the IEEE 754 standard for floating-point arithmetic, or the widespread availability of software for high-precision arithmetic.

${ }^{11}$ In [19], we used the notation $\mu(N)$ instead of $M(N)$.

${ }^{12}$ Here and elsewhere, "log" denotes the natural logarithm and "lg" denotes the logarithm to base 2. Note that, in [19], "log" denotes the logarithm to base 2 and "ln" is used for the natural logarithm.
} 
We also investigated $M(N)$ numerically and, as a result of the numerical evidence (see [19, Table II]), conjectured that

$$
\lim _{N \rightarrow \infty}\left(\frac{M(N) \lg \lg N}{N^{2}}\right)=1 .
$$

As numerical evidence for this conjecture, we found that, for $5 \leqslant n \leqslant 17$ and $N=2^{n}$, the following inequality holds:

$$
0.995<M(N) / M^{*}(N)<1.007, \text { where } M^{*}(N)=\frac{N^{2}}{0.71+\lg \lg N} .
$$

The constant 0.71 here was chosen empirically to give a good fit to the data; it does not affect the conjecture since $M^{*}(N) \sim N^{2} / \lg \lg N$ as $N \rightarrow \infty$.

Shortly after publication of [19], Paul Erdős, in a letter to the author, pointed out that the conjecture (11) is false, since it contradicts a result in a paper [26] that he published in 1960 (albeit in a rather inaccessible Russian journal). In fact, he showed that

$$
M(N)=\frac{N^{2}}{(\log N)^{c+o(1)}},
$$

where

$$
c=1-\frac{1+\ln \ln 2}{\ln 2} \approx 0.086
$$

is a small positive constant. Much later (in 2008), Ford [29, Corollary 3] gave the more precise result

$$
M(N) \asymp \frac{N^{2}}{(\log N)^{c}(\log \log N)^{3 / 2}} .
$$

Erdős's result (12) contradicts (11), since $\log N$ grows faster than any power of $\log \log N$ as $N \rightarrow \infty$. However, as the functions $(\log N)^{c}$ and $\log \log N$ grow very slowly, the true asymptotic rate of growth of $M(N)$ was not evident from computations with $N \leqslant 2^{17}$. For later computations with larger values of $N$, see [20].

We did, in some sense, have the last laugh, as it turned out that Erdős's proof of (12) in [26] was incorrect, as it used a known result outside its domain of applicability. This was pointed out by Karl Norton, and the proof was corrected in Erdős and Sárközy [27]. ${ }^{13}$

\subsection{Equivalence of some AGM algorithms}

We now describe an implicit error, where several authors made an implicit assumption that was later shown to be incorrect. Fortunately, this had no serious consequences.

The asymptotically fastest known algorithms for the high-precision computation of $\pi$ are based on the arithmetic-geometric mean (AGM) of Gauss and Legendre. The first such algorithm was discovered in 1976 by the author [13] and (independently) by Salamin [56]. It is sometimes called the Gauss-Legendre algorithm, since it is based on results that can be found in the work of these two mathematicians [16]. Subsequently, several other AGM-based algorithms were found by the Borwein brothers [10].

We give two of the AGM-based algorithms, the Gauss-Legendre algorithm (GL1), and the BorweinBorwein quartic algorithm (BB4) [10], as presented in [17, §4].

\footnotetext{
${ }^{13}$ Erdős and Sárközy [27] was published in 1980, so it is surprising that Erdős did not mention it to me in his letter of 1981. I only became aware of it forty years later, by following a chain of references on MathSciNet.
} 


\section{Algorithm GL1}

Input: The number of iterations $n_{\max }$.

Output: A sequence $\left(\pi_{n}^{\prime}\right)$ of approximations to $\pi$.

$$
\begin{aligned}
& a_{0}:=1 ; b_{0}:=1 / \sqrt{2} ; s_{0}:=\frac{1}{4} ; \\
& \text { for } n \text { from } 0 \text { to } n_{\max }-1 \text { do } \\
& a_{n+1}:=\left(a_{n}+b_{n}\right) / 2 ; \\
& c_{n+1}:=a_{n}-a_{n+1} ; \\
& \text { output } \pi_{n}^{\prime}:=a_{n+1}^{2} / s_{n} . \\
& \text { if } n<n_{\max }-1 \text { then } \\
& \quad b_{n+1}:=\sqrt{a_{n} b_{n}} \\
& s_{n+1}:=s_{n}-2^{n} c_{n+1}^{2} .
\end{aligned}
$$

Remark 6. Subscripts on variables such as $a_{n}, b_{n}$ are given for expository purposes. In an efficient implementation only a constant number of real variables are needed, because $a_{n+1}$ can overwrite $a_{n}$ (after saving $a_{n}$ in a temporary variable for use in the computation of $c_{n+1}$ ), and similarly for $b_{n}$, $c_{n}, s_{n}$, and $\pi_{n}^{\prime}$. Similar comments apply to Algorithm BB4.

\section{Algorithm BB4}

Input: The number of iterations $n_{\max }$.

Output: A sequence $\left(\pi_{n}^{\prime \prime}\right)$ of approximations to $\pi$.

$$
\begin{aligned}
& y_{0}:=\sqrt{2}-1 ; z_{0}:=2 y_{0}^{2} ; \\
& \text { for } n \text { from } 0 \text { to } n_{\text {max }}-1 \text { do } \\
& \text { output } \pi_{n}^{\prime \prime}:=1 / z_{n} ; \\
& \text { if } n<n_{\text {max }}-1 \text { then } \\
& \qquad \begin{aligned}
y_{n+1}:=\frac{1-\left(1-y_{n}^{4}\right)^{1 / 4}}{1+\left(1-y_{n}^{4}\right)^{1 / 4}} \\
\qquad z_{n+1}:=z_{n}\left(1+y_{n+1}\right)^{4}-2^{2 n+3} y_{n+1}\left(1+y_{n+1}+y_{n+1}^{2}\right)
\end{aligned}
\end{aligned}
$$

Algorithm GL1 produces a sequence $\left(\pi_{n}^{\prime}\right)$ of approximations to $\pi$. It is known that Algorithm GL1 has quadratic convergence, so (roughly speaking) the number of correct digits doubles at each iteration. More precisely, if $e_{n}^{\prime}:=\pi-\pi_{n}^{\prime}$ is the error in $\pi_{n}^{\prime}$, then

$$
0<e_{n}^{\prime}<\pi^{2} 2^{n+4} \exp \left(-2^{n+1} \pi\right) .
$$

Similarly, Algorithm BB4 produces a sequence $\left(\pi_{n}^{\prime \prime}\right)$ of approximations to $\pi$, and the sequence has quartic convergence, so (roughly speaking) the number of correct digits quadruples at each iteration. More precisely, if $e_{n}^{\prime \prime}:=\pi-\pi_{n}^{\prime \prime}$ is the error in $\pi_{n}^{\prime \prime}$, then

$$
0<e_{n}^{\prime \prime}<\pi^{2} 2^{2 n+4} \exp \left(-2^{2 n+1} \pi\right)
$$

In 2017-2018, we observed that the error bound on $e_{2 n}^{\prime}$, obtained from (14) by the substitution $n \rightarrow 2 n$, is exactly the same as the error bound on $e_{n}^{\prime \prime}$ given in (15). We computed some of the errors to high precision, and discovered that, not only were the error bounds equal, but the errors were identical. More precisely, $e_{n}^{\prime \prime}=e_{2 n}^{\prime}$ for all $n \geqslant 0$.

This implies that one iteration of Algorithm BB4 is equivalent to two iterations of Algorithm GL1, in the sense that $\pi_{n}^{\prime \prime}=\pi_{2 n}^{\prime}$. 
In the literature up to 2018, it had been (implicitly) assumed by the Borwein brothers, Bailey, Kanada, and others $[5,11,10,36]$ that the algorithms were inequivalent. ${ }^{14}$ For example, when computing $\pi$ to high precision, Kanada [36] used both algorithms as a consistency check. Although this would catch some programming errors, it does not provide an independent check that the constant computed is actually $\pi$. A better consistency check would be provided by a fast algorithm based on different theory, such as a Ramanujan-Sato series for $1 / \pi$, see [11], [17, §6]. The equivalence is implicit in the work of Guillera [33], but was not stated explicitly until 2018, when a proof was given in [17]. A more direct proof may be found in [46].

Remark 7. An iteration of Algorithm BB4 is about twice as time-consuming as an iteration of Algorithm GL1. On the other hand, Algorithm GL1 requires twice as many iterations to obtain the same precision. Thus, it is not clear which algorithm is preferable in practice. For more on this topic, see [17, end of §4].

\section{Some conclusions}

There are many classes of mathematical errors. We list some of the more common ones. An awareness of such errors may help the reader to avoid similar ones.

(1) Numerical or algebraic errors, possibly caused by an incorrect or numerically unstable algorithm, or an error in its implementation as a computer program.

(2) Errors of omission. For example, Poincaré missed the possibility of chaotic behaviour (§2.3), and Vassilev-Missana failed to consider integers with more than two distinct prime factors $(\S 3.1)$.

(3) Unwarranted assumption, such as in our analysis of the binary Euclidean algorithm $(\S 4.1)$. Many incorrect proofs of $\mathrm{RH}$ are in this class. For example, they may assume that some property of $\zeta(s)$ that holds for $\mathfrak{R}(s)>1$ also holds for $1 / 2 \leqslant \mathfrak{R}(s) \leqslant 1$.

(4) Gap in the proof. A recent example is Wiles's first proof of FLT, see §2.5. More generally, perhaps the author proves A and claims that A implies B, but the implication is not obvious it may be true but needs to be proved. Also, a proof may be logically correct, so far as it goes, but not prove what is claimed. For example, if we are trying to prove a statement $(\forall n \geqslant 0) P(n)$, it is not (usually) sufficient to prove $P(0), P(1), \ldots, P(99)$.

(5) Using an incorrect result from a published paper. For example, Agélas (§3) used an incorrect result of Vassilev-Missana. In the worst case this could lead to a whole tree of incorrect results.

(6) Using a correct result but applying it incorrectly. For example, the definitions may be subtly different, or the domain of applicability of the correct result may not be taken into account correctly. See for example the discussion of Erdős [26, 27] in §4.2.

(7) General lack of clarity or rigor, so although the proof may be correct in some sense, it is not currently accepted by the mathematical community (see §2.6). Old examples include many of Euler's proofs, and various proofs of the fundamental theorem of algebra that were not rigorous by modern standards. More recently, some well-known examples are from the Italian school of algebraic geometry [69] in the period 1885-1935.

\section{Acknowledgements}

$\S 3$ is a summary of material in [18]. Kannan Soundararajan pointed out some relevant discussion on MathOverflow [41]. We thank Léo Agélas for his correspondence regarding §3, and both Léo Agélas and Artur Kawalec for confirming some of the computations in §3.

\footnotetext{
${ }^{14}$ The equivalence result assumes that arithmetic is exact. If, as is necessary in practice, limited-precision approximations are used, then $\pi_{n}^{\prime \prime}$ and $\pi_{2 n}^{\prime}$ may differ slightly, due to the effect of rounding errors.
} 
We thank Philippe Flajolet ${ }^{15}$, Don Knuth, and Brigitte Vallée, for their correspondence regarding §4.1, and Paul Erdős ${ }^{16}$, Donald J. Newman ${ }^{17}$, Andrew Odlyzko, and Carl Pomerance, for correspondence regarding the conjecture of $\S 4.2$. We also thank Jonathan Borwein ${ }^{18}$ and David Bailey for discussions regarding AGM-based algorithms relevant to §4.3. Finally, thanks to Rob Corless and Nick Trefethen for their comments on the first draft of this paper, and for noticing some typos.

\section{References}

[1] Léo Agélas, Generalized Riemann Hypothesis, 29 May 2019, hal-00747680v3. https://hal.archives-ouvertes. fr/hal-00747680v3.

[2] Anon, Science: As you were, Time XLV, 18, April 30, 1945. http://content.time.com/time/subscriber/ article/0,33009,797441,00.html.

[3] Kenneth Appel and Wolfgang Haken, Every planar map is four colorable. I. Discharging, Illinois f. Math. 21, 3 (1977), 429-490. Also part II, ibid, 491-567.

[4] Kenneth Appel and Wolfgang Haken, Every Planar Map is Four-Colorable, Contemporary Mathematics 98, Amer. Math. Soc., Providence, Rhode Island, 1989.

[5] David H. Bailey, The computation of $\pi$ to $29,360,000$ decimal digits using Borweins' quartically convergent algorithm, Math. Comp. 50 (1988), 283-296.

[6] Peter Ball, Proof claimed for deep connection between primes, Nature, 10 Sept. 2012. https://doi.org/10. 1038/nature.2012.11378

[7] June Barrow-Green, Poincaré and the Three Body Problem, American Math. Soc., Rhode Island, 1997.

[8] Bruce C. Berndt, Hans Rademacher (1892-1969), Acta Arithmetica LXI.3 (1992), 209-231.

[9] Jonathan M. Borwein and David H. Bailey, Mathematics by Experiment: Plausible Reasoning in the 21st Century, 2nd edition, A. K. Peters, Wellesley, Massachusetts, 2008.

[10] Jonathan M. Borwein and Peter B. Borwein, Pi and the AGM: A Study in Analytic Number Theory and Computational Complexity, Monographies et Études de la Société Mathématique du Canada, John Wiley \& Sons, Toronto, 1987.

[11] Jonathan M. Borwein, Peter B. Borwein, and David H. Bailey, Ramanujan, modular equations, and approximations to pi or how to compute one billion digits of pi, Amer. Math. Monthly 96 (1989), 201-219.

[12] Peter Borwein, Stephen Choi, Brendan Rooney, and Andrea Weirathmueller (eds.), The Riemann Hypothesis, CMS Books in Mathematics, Vol. 27, Springer, New York, 2008.

[13] Richard P. Brent, Fast multiple-precision evaluation of elementary functions, f. ACM 23 (1976), 242-251.

[14] Richard P. Brent, Analysis of the binary Euclidean algorithm, New Directions and Recent Results in Algorithms and Complexity (edited by J. F. Traub), Academic Press, New York, 1976, 321-355. Errata: https://maths-people.anu.edu.au/ brent/pub/pub037.html.

[15] Richard P. Brent, Twenty years' analysis of the binary Euclidean algorithm, Millennial Perspectives in Computer Science: Proceedings of the 1999 Oxford-Microsoft Symposium in honour of Sir Tony Hoare (edited by J. Davies et al), Palgrave, New York, 2000, 41-53. Also (extended version) https://arxiv.org/abs/1303.2772, 2013.

[16] Richard P. Brent, Old and new algorithms for $\pi$, Notices of the AMS (letter to the editor) 60, 1 (Jan. 2013), 7. Also https://arxiv.org/abs/1303.2762.

[17] Richard P. Brent, The Borwein brothers, pi and the AGM, From Analysis to Visualisation: A Celebration of the Life and Legacy of Jonathan M. Borwein (Brailey Sims et al, editors), Springer Proceedings in Mathematics and Statistics 313 (2020), 323-348. Also https://arxiv.org/abs/1802.07558, 2018. Proceedings errata: https://maths-people.anu.edu.au/ brent/pub/JBCC-errata.html.

[18] Richard P. Brent, On some results of Agélas concerning the GRH and of Vassilev-Missana concerning the prime zeta function, https://arxiv.org/abs/2103.09418, 2021.

\footnotetext{
${ }^{15}$ Philippe Flajolet 1948-2011.

${ }^{16}$ Paul Erdős 1913-1996.

${ }^{17}$ Donald J. Newman 1930-2007.

${ }^{18}$ Jonathan Borwein 1951-2016.
} 
[19] Richard P. Brent and H. T. Kung, The area-time complexity of binary multiplication, f. ACM 28 (1981), 521-534. Corrigendum: ibid 29 (1982), 904. Also https://maths-people.anu.edu.au/ brent/pub/pub055.html.

[20] Richard P. Brent, Carl Pomerance, David Purdum, and Jonathan Webster, Algorithms for the multiplication table, https://arxiv.org/abs/1908.04251, 2021.

[21] Christophe Breuil, Brian Conrad, Fred Diamond, and Richard Taylor, On the modularity of elliptic curves over Q: wild 3-adic exercises, f. Amer. Math. Soc. 14, 4 (2001), 843-939.

[22] Davide Castelvecchi, Mathematical proof that rocked number theory will be published, Nature 580, 3 April 2020, 177. https://www.nature.com/articles/d41586-020-00998-2.

[23] Winston Churchill, Speech to the House of Commons, 1948 (paraphrasing Santayana [57, Vol. 1, Ch. 12]).

[24] Harold Davenport, Multiplicative Number Theory, Graduate Texts in Mathematics, Vol. 74 (third edition, revised and with a preface by Hugh L. Montgomery), Springer-Verlag, NY, 2000.

[25] Florin Diacu, The solution of the $n$-body problem, The Mathematical Intelligencer 18, 3 (1996), 66-70.

[26] Paul Erdős, An asymptotic inequality in the theory of numbers, Vestnik Leningrad Univ. 15, Ser. Mat. Mekh. Astron. No. 3, 13 (1960), 41-49 (in Russian). MR0126424 (23\#A3720), Zbl 0104.26804.

[27] Paul Erdős and András Sárközy, On the number of prime factors in integers, Acta Sci. Math. (Szeged) 42, 3-4 (1980), 237-246. MR603312 (82c:10053).

[28] Philippe Flajolet and Robert Sedgewick, Analytic Combinatorics, Cambridge Univ. Press, 2009.

[29] Kevin Ford, The distribution of integers with a divisor in a given interval, Annals of Math. (2) 168 (2), 2008, 367-433.

[30] Carl-Eric Fröberg, On the prime zeta function, BIT Numerical Mathematics (formerly BIT) 8 (1968), 187-202.

[31] Jeremy Gray, Poincaré in the archives - two examples, Philosophia Scientiæ 2, 3 (1997), 27-39. http: //www.numdam.org/article/PHSC_1997_2_3_27_0.pdf.

[32] Jeremy Gray, Henri Poincaré: a Scientific Biography, Princeton University Press, Princeton, 2013.

[33] Jesús Guillera, Easy proofs of some Borwein algorithms for $\pi$, Amer. Math. Monthly 115 (2008), 850-854.

[34] Adolf J. Hildebrand, Introduction to Analytic Number Theory, Math 531 Lecture Notes, Fall 2005. https: //faculty.math.illinois.edu/ hildebr/ant/main.pdf.

[35] Georges Ifrah, The Universal History of Numbers: From Prehistory to the Invention of the Computer, John Wiley \& Sons, New York, 2000.

[36] Yasumasa Kanada, Vectorization of multiple-precision arithmetic program and 201, 326, 000 decimal digits of pi calculation, Supercomputing 88, IEEE, 1988, 117-128. https://doi.org/10.1109/SUPERC.1988.74139

[37] Alfred B. Kempe, On the geographical problem of four colours, Amer. F. of Math. 2 (1879), 193-220.

[38] Erica Klarreich, Titans of mathematics clash over epic proof of ABC conjecture, Quanta Magazine, Sept. 20, 2018.

[39] Donald E. Knuth, The Art of Computer Programming, Vol. 2, 3rd edition, Addison-Wesley, Menlo Park, Ca., 1998.

[40] David W. Masser, Open problems, Proceedings of a Symposium on Analytic Number Theory (W. Chen, ed.), Imperial College, London, 1985.

[41] Mathoverflow, Question by user Klangen and comments/answers at https://mathoverflow.net/questions/ 288847/, 2017-2021.

[42] Mathoverflow, Question by user TKZim and answers by Carlo Beenakker and Todd Trimble at https: //mathoverflow.net/questions/293545/, 2018.

[43] Jean Mawhin, Henri Poincaré: a life in the service of science, Notices of the AMS 52, 9 (2005), 1036-1044.

[44] Gérard Maze, Existence of a limiting distribution for the binary GCD algorithm, f. Discrete Algorithms 5 (2007), 176-186.

[45] Franz Mertens, Über eine zahlentheoretische Function”, Sitzungsberichte Akad. Wien 106, Abt. 2a (1897), 761-830.

[46] Lorenz Milla, Easy proof of three recursive $\pi$-algorithms, https://arxiv.org/abs/1907.04110, 2019.

[47] Ian D. Morris, A rigorous version of R. P. Brent's model for the binary Euclidean algorithm, Advances in Mathematics 290 (2016), 73-143. https://doi.org/10.1016/j.aim.2015.12.008

[48] John J. O'Connor and Edmund F. Robertson, Lars Edvard Phragmén, MacTutor History of Mathematics Archive, https://mathshistory.st-andrews.ac.uk/Biographies/Phragmen/, 2011. 
[49] John J. O'Connor and Edmund F. Robertson, Jules Henri Poincaré, MacTutor History of Mathematics Archive, https://mathshistory.st-andrews.ac.uk/Biographies/Poincare/, 2003.

[50] Andrew M. Odlyzko and Herman J. J. te Riele, Disproof of the Mertens conjecture, f. für die reine und angewandte Mathematik 357 (1985), 138-160.

[51] Joseph Oesterlé, Nouvelles approches du "théorème" de Fermat, Séminaire Bourbaki, Vol. 1987/88. Astérisque 161-162 (1988), Exp. No. 694, 4 (1989), 165-186. MR0992208 (90g:11038)

[52] Max K. Planck, Scientific Autobiography and Other Papers, Philosophical Library, New York, 1950.

[53] Henri Poincaré, Sur le problème des trois corps et les équations de la dynamique avec des notes par l'auteurmémoire couronné du prix de S. M. le Roi Oscar II, 1889. Printed for Acta Math. but withdrawn.

[54] Henri Poincaré, Sur le problème des trois corps et les équations de la dynamique, Acta Math. 13, 1890, $1-270$.

[55] Karl Sabbagh, The Riemann Hypothesis: the Greatest Unsolved Problem in Mathematics, Farrar, Straus and Giroux, 2004.

[56] Eugene Salamin, Computation of $\pi$ using arithmetic-geometric mean, Math. Comp. 30 (1976), 565-570.

[57] George Santayana, "Those who cannot remember the past are condemned to repeat it", The Life of Reason, 1905. http://www.gutenberg.org/files/15000/.

[58] Peter Scholze and Jakob Stix, Why $a b c$ is still a conjecture, manuscript, 2018. Available from https: //ncatlab.org/nlab/files/why_abc_is_still_a_conjecture.pdf.

[59] Simon Singh, Fermat's Last Theorem, William Collins, London, 1997.

[60] Thomas J. Stieltjes, Lettre à Hermite de 11 Juillet 1885, Lettre \#79, in B. Baillaud and H. Bourget (eds.), Correspondance d'Hermite et Stieltjes, Gauthier-Villars, Paris, 1905, 160-164.

[61] Peter Guthrie Tait, Remarks on the previous communication, Proc. R. Soc. Edinburgh 10 (1880), 729.

[62] Richard Taylor and Andrew Wiles, Ring theoretic properties of certain Hecke algebras, Annals of Math. (2) 141 (3), 1995, 553-572.

[63] E. C. Titchmarsh, The Theory of the Riemann Zeta-function, 2nd edition (edited and with a preface by D. R. Heath-Brown), Oxford, 1986.

[64] Brigitte Vallée, Dynamics of the binary Euclidean algorithm: functional analysis and operators, Algorithmica 22 (1998), 660-685.

[65] Mladen Vassilev-Missana, A note on prime zeta function and Riemann zeta function, Notes on Number Theory and Discrete Mathematics 22, 4 (2016), 12-15. Corrigendum ibid 27, 2 (2021), to appear.

[66] Wikipedia, abc conjecture, https://en.wikipedia.org/wiki/Abc_conjecture.

[67] Wikipedia, Fermat's last theorem, https://en.wikipedia.org/wiki/Fermat\%27s_Last_Theorem\#Prizes_and_ incorrect_proofs.

[68] Wikipedia, Four color theorem, https://en.wikipedia.org/wiki/Four_color_theorem\#Early_proof_attempts.

[69] Wikipedia, Italian school of algebraic geometry, https://en.wikipedia.org/wiki/Italian_school_of_algebraic_ geometry.

[70] Wikipedia, List of incomplete proofs. https://en.wikipedia.org/wiki/List_of_incomplete_proofs.

[71] Wikipedia, Mertens conjecture. https://en.wikipedia.org/wiki/Mertens_conjecture.

[72] Wikipedia, Szpiro's conjecture, https://en.wikipedia.org/wiki/Szpiro\%27s_conjecture.

[73] Wikipedia, Wiles's proof of Fermat's Last Theorem https://en.wikipedia.org/wiki/Wiles\%27s_proof_of Fermat\%27s_Last_Theorem

[74] Andrew Wiles, Modular elliptic curves and Fermat's Last Theorem, Annals of Math. (2) 141 (3), 1995, 443-551.

[75] Peter Woit, $\mathrm{ABC}$ is still a conjecture, March 4, 2021. https://www.math.columbia.edu/ woit/wordpress/ $? \mathrm{p}=12220$. 